\title{
Preliminary results of the cosmic ray study in the NUCLEON space experiment
}

\section{L.Tkachev ${ }^{1}$}

Joint Institute for Nuclear Research, Dubna, 141980, Russia

E-mail: tkatchev@jinr.ru

N.Gorbunov, V.Grebenyuk, S.Porokhovoy, A.Sadovsky

Joint Institute for Nuclear Research, Dubna, 141980, Russia

\section{A.Tkachenko}

Joint Institute for Nuclear Research, Dubna, 141980, Russia

Bogolyubov Institute for Theoretical Physics, Kiev, Ukrain

\section{E.Atkin, V.Shumikhin}

National Research Nuclear University “MEPhI”, Moscow, 115409, Russia

\section{V.Bulatov, V.Dorokhov, S.Filippov, D.Polkov}

SDB Automatica, Ekaterinburg, 620075, Russia

D.Karmanov, I.Kovalev, I.Kudryashov, M.Merkin, A.Pakhomov, A.Panov, D.Podorozhny, L.Sveshnikova, M.Torochkov , A.Turundaevskiy, O.Vasiliev,

\section{A.Voronin}

Skobeltsyn Institute of Nuclear Physics, Moscow State University, Moscow, 119991, Russia

The orbital NUCLEON detector was launched at 26 December 2014 for 5 years of data taken. The experiment is designed to measure cosmic ray energy spectrum and charge composition at $100 \mathrm{GeV}-1000 \mathrm{TeV}$ and $\mathrm{Z}=1-30$ respectively. The NUCLEON apparatus structure, methods of primary cosmic ray charge and energy measurements are described. Preliminary results of the measurements of the cosmic nuclei energy spectra and the charge composition are presented from the first year and a half of data taking from orbit.

38th International Conference on High Energy Physics

3-10 August 2016

Chicago, USA

\footnotetext{
${ }^{1}$ Speaker

(C) Copyright owned by the author(s) under the terms of the Creative Commons

Attribution-NonCommercial-NoDerivatives 4.0 International License (CC BY-NC-ND 4.0).
} 


\section{The NUCLEON experiment aims and its design}

The NUCLEON satellite experiment is designed to investigate directly the energy spectra of cosmic ray (CR) nuclei and the CR composition before the "knee" from $100 \mathrm{GeV}$ to $1000 \mathrm{TeV}$. The additional aim is the $\mathrm{CR}$ electron spectrum measurement from $20 \mathrm{GeV}$ to $3 \mathrm{TeV}$. The "knee" energy range, $10^{14}-10^{16} \mathrm{eV}$, is a crucial region for the understanding of the $\mathrm{CR}$ acceleration and propagation in the interstellar medium. It is important to obtain more data with elemental resolution since the "knee" region is interesting for astrophysics.

The NUCLEON device was designed and produced by a collaboration of SINP MSU (main investigator), JINR (Dubna) and some other Russian scientific and industrial centers. It is placed as an additional payload on board of the RESURS-P №2 satellite (left of Figure 1) that was launched at 26 December 2014. The spacecraft has a sun-synchronous orbit with an inclination of $\sim 97$ degrees and a middle altitude of $\sim 475 \mathrm{~km}$.

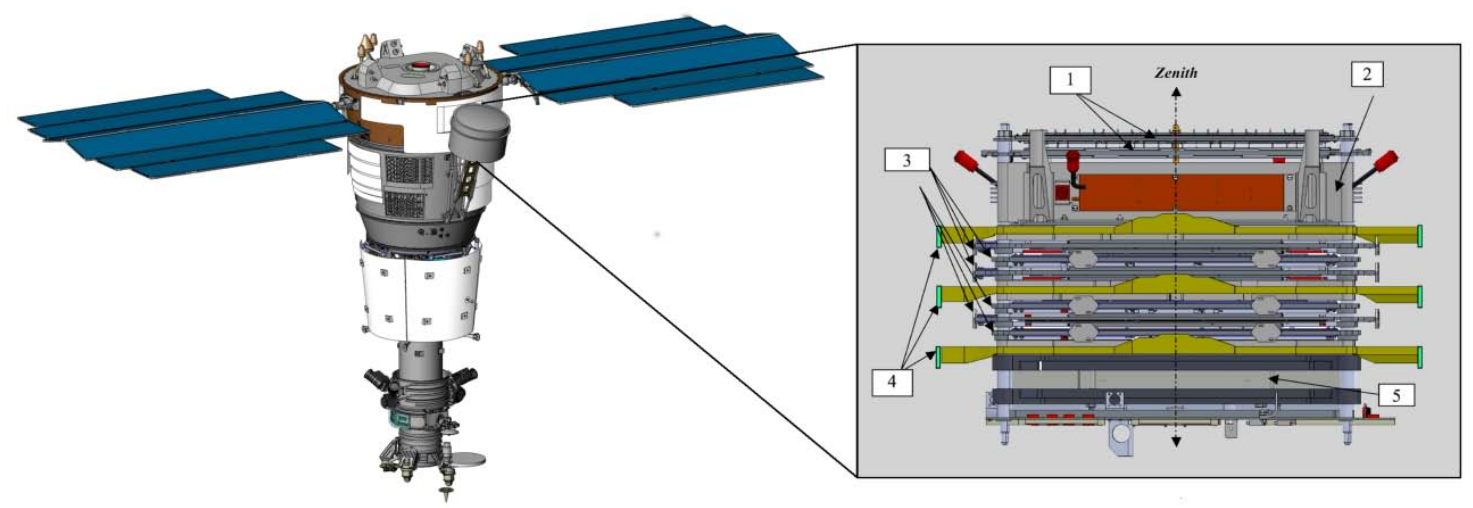

Figure 1. Left: the RESURS-P №2 satellite with the NUCLEON detector. Right: the simplified layout of NUCLEON experiment detectors.

The simplified layout of NUCLEON experiment is presented in right of Figure 1: (1) 4 planes of the silicon pads for primary particle charge measurement; (2) - carbon target; (3) - 6 silicon microstrip planes of the energy measurement system utilizing the KLEM technique; (4) 3 double scintillator planes of the trigger system; (5) -6 silicon microstrip planes divided by tungsten absorber planes of the calorimeter(MIK) with the total depth $\sim 16$ radiation lengths; more than 10000 electronic channels in total.

The primary energy is measured by registration of spatial density of the secondary particles with the KLEM (Kinematic Lightweight Energy Meter) method [1] and/or with the calorimeter (MIK). The effective geometric factor is more than $0.2 \mathrm{~m}^{2} \mathrm{sr}$ for the KLEM and near $0.06 \mathrm{~m}^{2} \mathrm{sr}$ for the calorimeter. The Monte-Carlo simulation shows that accuracy of energy measurement with the KLEM procedure is $\sim 60 \%$ for energies $\mathrm{E}>1 \mathrm{TeV}$, which is confirmed by the results of tests at CERN SPS beams. The charge measurement system was tested at CERN SPS and provide the charge resolution $\Delta \mathrm{Z} / \mathrm{Z}$ better than 0.3 . The NUCLEON statistics obtained for a year and a half data taking is about $1.5 \cdot 10^{7}$ triggers.

\section{The NUCLEON experiment preliminary results}

The preliminary results of the CR study at the NUCLEON experiment is presented in Figures 2 - 4 in comparison with the corresponding measurements of other experiments: ATIC [2], JACEE [3], SOKOL [4], CREAM [5], TRACER [6] and AMS-2 [7]. The NUCLEON data 
have the large systematic uncertainties in the low energy part of the CR spectra due to trigger system suppression of such events. The different trigger effects are under investigation presently with Monte-Carlo simulation. This is the reason that NUCLEON results are given for energies starting with $\mathrm{E}>3 \mathrm{TeV}$ where the trigger system efficiency is $\sim 100 \%$.
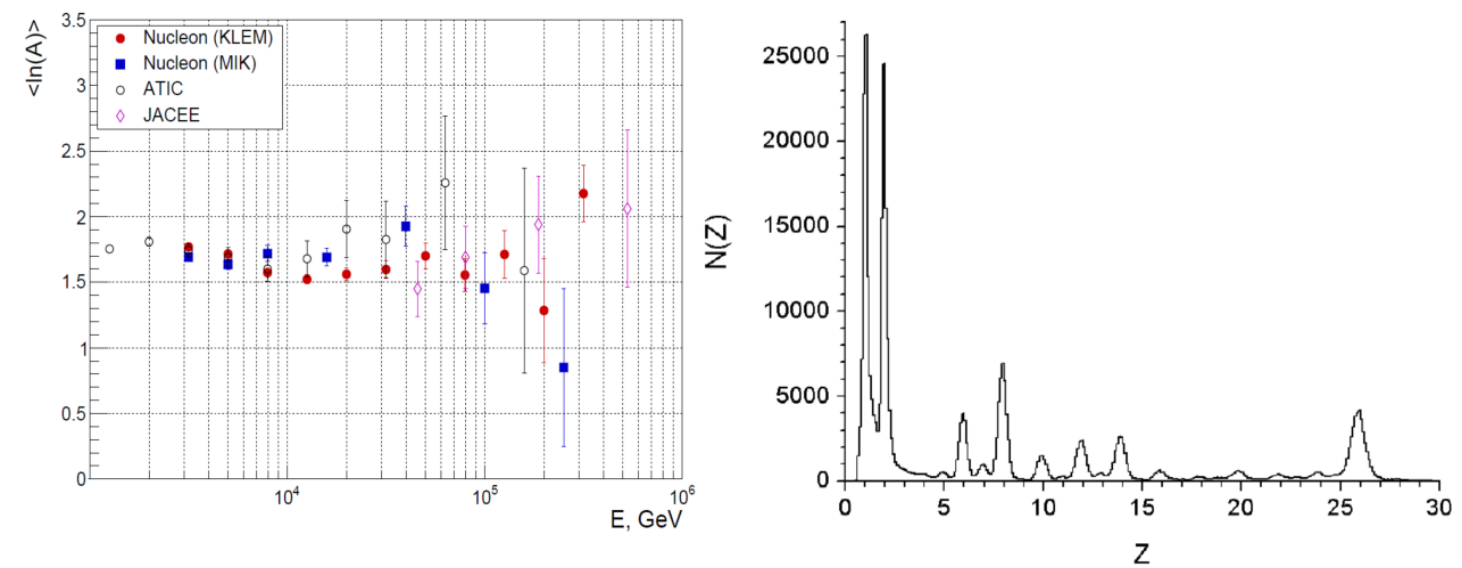

Figure 2. Left: the $C R<\ln (A)>$ dependence of energy. Right: the different CR nuclei abundance.

As it followed from the $\mathrm{CR}<\ln (\mathrm{A})>$ dependence of energy presented in the left of Figure 2 there is reasonable accordance between NUCLEON data and of the ATIC[2] and JACEE[3] data at the present level of available statistics. The difference between the NUCLEON data that was obtained with KLEM or calorimeter measurements may be considered approximately as the existing systematical uncertainties. At right of the Figure 2 the different CR nuclei abundance is shown as it measured by the NUCLEON charge measurement system. Besides of proton and He peaks the $\mathrm{C}, \mathrm{O}$, Fe and some other nuclei peaks are clearly seen. Relative values of proton and He peaks are essentially modified by the trigger system event selection.

In the Figure 3 the preliminary results of the $\mathrm{CR}$ proton and He nuclei spectra measurements are presented in comparison with the ATIC[2], SOCOL[4], CREAM[5], TRACER[6] and AMS-2[7,8] data. Similarly, in the Figure 4 the preliminary results of the CR $\mathrm{C}, \mathrm{O}$ and $\mathrm{Fe}$ nuclei spectra measurements are presented. Besides, in the bottom right panel the $\mathrm{p} /$ He ratio dependence of energy is presented. Everywhere the NUCLEON results are shown for KLEM and calorimeter approach measurements separately and their reasonable accordance to the other experimental data is obtained.
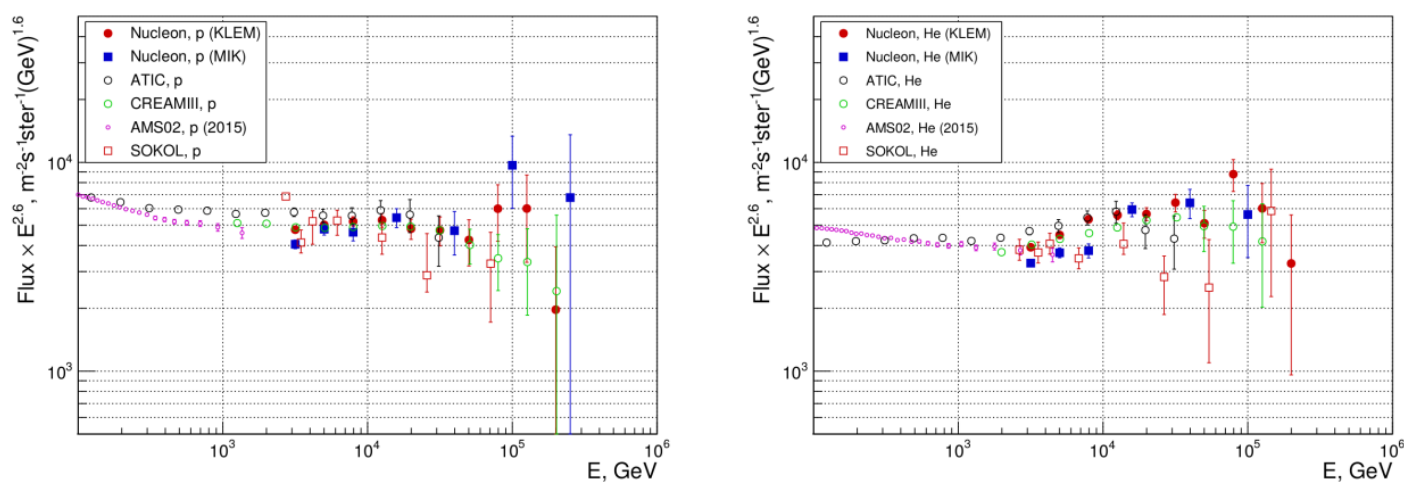

Figure 3. Preliminary results of the CR proton and helium spectra measurements. 

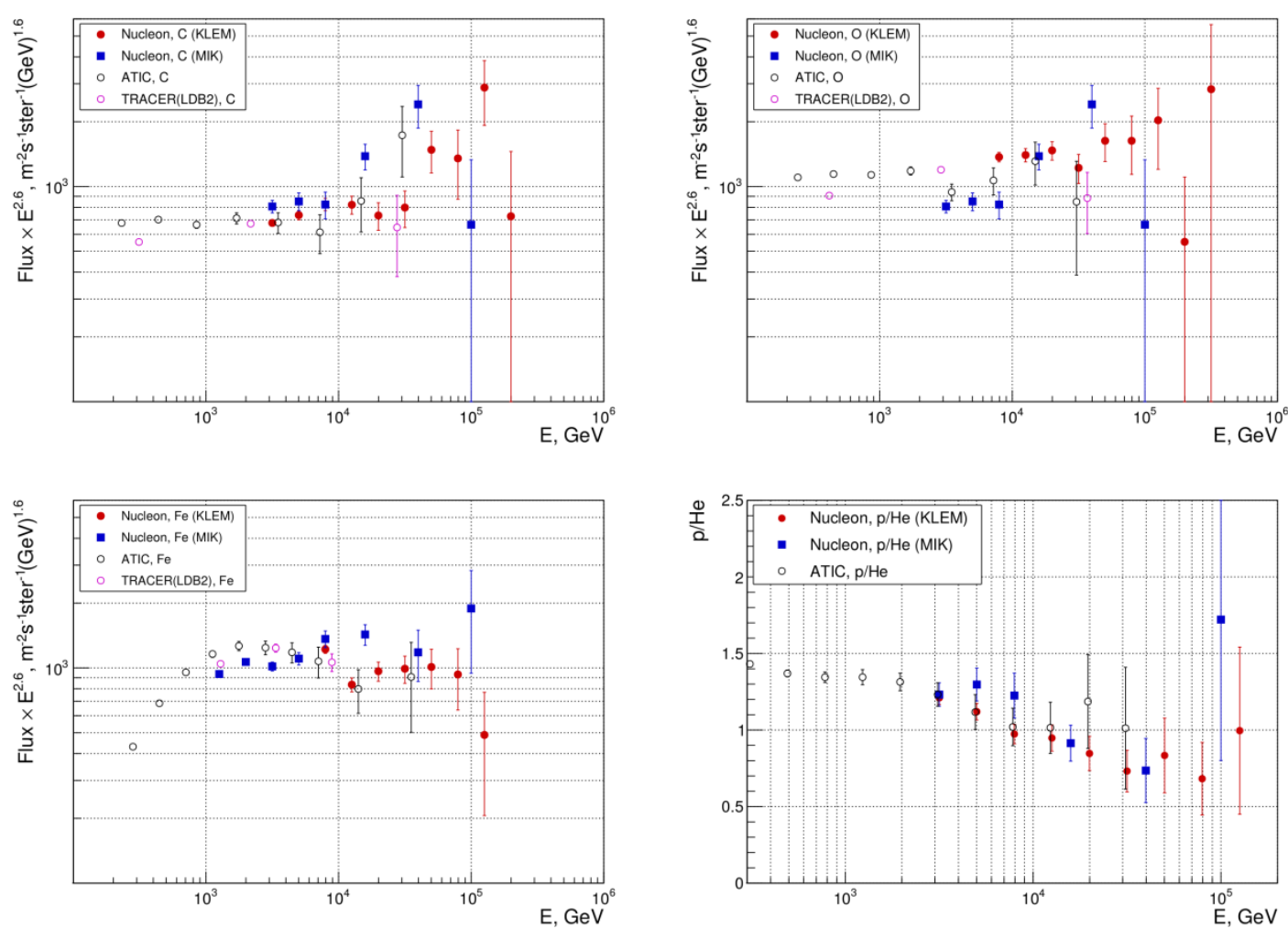

Figure 4. Preliminary results of the CR C, O and Fe nuclei spectra and p/He ratio measurements

\section{References}

[1] G. L. Bashindzhagyan et al., A new method for determining particle energy in the range $10^{11}-10^{15} \mathrm{eV}$ and results from a beam test at $180 \mathrm{GeV/c}$. Instruments and experimental techniques. 48(2005)3236.

[2] A. D. Panov, J. H. Adams Jr. et al., Energy spectra of abundant nuclei of primary cosmic ray from the data of ATIC-2 experiment, Bulletin of the Russian academy of science: physics, 73(2009)564567.

[3] Y. Takahashi (for the JACEE Collaboration), Elemental abundance of high energy cosmic ray, Nucl.Phys. B. In proceeding Suppl.,60(1998).

[4] I. P. Ivanenko et al., Energy spectra of cosmic rays above $2 \mathrm{TeV}$ as measured by the "SOKOL" apparatu ,In proceeding of $23^{\text {rd }}$ ICRC, Calgary, Canada, 2(1993)1719

[5] S. Yoon et al., Preliminary proton and He spectra from the CREAM-III flight. In proc.31 ${ }^{\text {st }}$ ICRC(2009), ID0668

[6] A. Obermeier et al., Energy spectra and seconraty CR nuclei measured with TRACER. ApJ 14(2011)742.

[7] M. Aguilar et al., Precision measurement of the proton flux in primary CR from rigidity $1 G V$ to 3 TV with AMS on ISS.,PRL 114(2015)171103.

[8] M. Aguilar et al., Precision measurement of the He flux in primary CR of rigidities 1.9 GV to 3 TV with AMS on ISS. PRL 112(2015)21101. 